\title{
Impulsando la Educación Abierta en Latinoamérica desde la Comunidad GeoGebra Latinoamericana
}

\author{
Impulsionando a educação aberta na América Latina a partir da
} Comunidade Latino-Americana de GeoGebra

\author{
Boosting Open Education in Latin America from the \\ Latin American GeoGebra Community
}

\section{SERGIO RUBIO-PIZZORNO ${ }^{1}$}

\author{
0000-0003-3624-1829 iD \\ researchgate.net/profile/Sergio_Rubio-Pizzorno $\mathrm{R}^{6}$ \\ geogebra.org/u/zergiorubiob?
}

http://dx.doi.org/10.23925/2237-9657.2020.v9i1p10-25

\section{RESUMEN}

Según la Declaración de Panamá de 2108, la Educación Abierta es uno de los elementos esenciales para el desarrollo de la Ciencia Abierta en Latinoamérica. Bajo esta premisa, en este artículo se propone una ampliación al modelo de Educación Abierta presentado en tal declaración, para pasar de lo únicamente técnico y pragmático, a incorporar una dimensión social integradora: lo comunitario.

Para construir este modelo ampliado se realiza una revisión de los aspectos que lo componen (recursos educativos abiertos, prácticas educativas abiertas y comunidades educativas abiertas), los cuales se ilustran a través de los aportes y desarrollos de GeoGebra. Específicamente se centra la atención en el Coloquio GeoGebra, un proyecto de la Comunidad GeoGebra Latinoamericana, con la intención de mostrar un ejemplo concreto de la articulación de los tres aspectos del modelo de Educación Abierta aquí propuestos.

Palabras claves: Educación Abierta; Comunidad GeoGebra Latinoamericana; Coloquio GeoGebra

\section{RESUMO}

De acordo com a Declaração do Panamá de 2108, a Educação Aberta é um dos elementos essenciais para o desenvolvimento da ciência aberta na América Latina. Sob essa premissa, este artigo propõe uma extensão do modelo de Educação Aberta apresentado nesta declaração, para passar do meramente técnico e pragmático, para incorporar uma dimensão social integradora: a comunidade.

\footnotetext{
${ }^{1}$ Instituto GeoGebra Internacional y Comunidad GeoGebra Latinoamericana - $\underline{\text { sergio.rubio@ cinvestav.mx }}$
} 
Para construir esse modelo, é feita uma revisão dos aspectos que o compõem (recursos educacionais abertos, práticas educacionais abertas e comunidades educacionais abertas), ilustrados pelas contribuições e desenvolvimentos do GeoGebra. Especificamente, a atenção está voltada para o Colóquio GeoGebra, um projeto da Comunidade GeoGebra da América Latina, com a intenção de mostrar um exemplo concreto da articulação dos três aspectos do modelo de Educação Aberta proposto aqui.

Palavras-chave: Educação aberta; Comunidade Latino-Americana de GeoGebra; Colóquio GeoGebra

\begin{abstract}
According to the Panama Declaration of 2108, Open Education is one of the essential elements for the development of Open Science in Latin America. Under this premise, this article proposes an extension of the Open Education model presented in that declaration, to move from the merely technical and pragmatic, to incorporate an integrating social dimension: the community.

In order to build this expanded model, a review of the aspects that make it up (open educational resources, open educational practices and open educational communities) is carried out, which are illustrated through the contributions and developments of GeoGebra. Specifically, attention is focused on the GeoGebra Colloquium, a project of the Latin American GeoGebra Community, with the intention of showing a concrete example of the articulation of the three aspects of the Open Education model proposed here.
\end{abstract}

Keywords: Open Education; Latin American GeoGebra Community; GeoGebra Colloquium.

\title{
Introducción
}

GeoGebra ha vivido una evolución tecnológica y conceptual desde su creación, acorde a su filosofía libre y abierta. En un primer momento apareció el software, el cual fue usado principalmente por profesoras y profesores de matemáticas para crear recursos educativos. Estos recursos se compartieron de manera abierta con la red global de usuarios de GeoGebra, quienes podían usar y adaptar tales recursos libremente. La oportunidad de crear recursos educativos y compartirlos de manera abierta dio paso al desarrollo y consolidación de la configuración más social de GeoGebra, es decir, su comunidad.

La evolución de GeoGebra también se ha ido construyendo en y desde Latinoamérica, en donde desde hace algunos años un grupo personas entusiastas de GeoGebra y la Educación Abierta se han comenzado a organizar persiguiendo los objetivos de "visibilizar los aportes realizados en nuestra región, y explorar opciones que permitan una permanente articulación de la Comunidad" (Rubio-Pizzorno, León Salinas, León Ríos, Córdoba-Gómez y Abar, 2018, p. 1919). De ahí que este grupo se identifique con el nombre de Comunidad GeoGebra Latinoamericana (CGL), por su compromiso con el desarrollo de la Comunidad GeoGebra en y desde Latinoamérica.

A modo de ilustración de este compromiso y el impulso a la Educación Abierta por parte de la CGL, en el presente artículo se aborda uno de sus proyectos, el Coloquio GeoGebra, y en particular su primer año de vida. Esto permitirá mostrar la influencia de ciertos elementos de la Educación Abierta (como los recursos y las prácticas) en la constitución y desarrollo de una comunidad educativa abierta (la CGL). 


\section{Contexto general: Educación Abierta}

A finales de 2018, un grupo de científicas y científicos latinoamericanos se reunieron en Panamá para elaborar una declaración sobre la Ciencia Abierta en nuestra región, con recomendaciones para trabajar por este objetivo bajo la premisa de que el conocimiento es un bien común (Stacey y Hinchliff Pearson, 2017). Como resultado declararon 10 elementos esenciales de la Ciencia Abierta: el Acceso Abierto, los Datos Abiertos de investigación, la Educación Abierta, la Ciencia ciudadana, la Investigación abierta, reproducible y replicable, la Evaluación Abierta, las Herramientas Abiertas: Política de software y hardware libres, las Infraestructuras Abiertas, la Innovación de Código Abierto y las Licencias Libres (Declaración de Panamá, 2018).

Estos elementos esenciales se han ido construyendo a lo largo de la historia reciente de formas diversas e incluyentes, aunque con un detonador común: la aparición de las tecnologías digitales en el panorama de la humanidad o también llamada revolución digital (Stacey y Hinchliff Pearson, 2017). Si bien han existido experiencias abiertas antes de este hito, como por ejemplo "en la década de 1970 para tratar prácticas específicas en la educación infantil, o para describir las prácticas de las universidades abiertas" (Santos, 2013, pp. 86 y 87), la aparición de las tecnologías digitales sirvió de catalizador social para visibilizar y reconocer fenómenos culturales que necesitaban ser revisados y resignificados. Entre ellos se encuentra la manera de hacer y publicar la ciencia (Aguado-López, Becerril-García y ChávezÁvila, 2019), la manera de producir y desarrollar hardware y software (Free Software Foundation, 2020), la disponibilidad de recursos educativos o la posibilidad de crearlos personalmente (Santana, Rossini y Pretto, 2013), y en definitiva, gracias a la aparición de las tecnologías digitales, las personas han tenido "la posibilidad [...] de materializar las soluciones a las problemáticas y hacerlo de manera colaborativa" (Rubio-Pizzorno, 2018, p. 52), para atender necesidades personales, grupales y comunitarias.

En el caso de la Educación Abierta y de la manera en que se ha ido construyendo a lo largo de la historia reciente, en este artículo se describe una ruta de su evolución -reconociendo que se pueden delinear otras igualmente válidas-, la cual transita por aspectos tecnológicos, pragmáticos y comunitarios, específicamente referidos a los Recursos Educativos Abiertos (REA), las Prácticas Educativas Abiertas (PEA) y las Comunidades Educativas Abiertas (CEA) respectivamente. La manifestación de estos aspectos en la evolución de GeoGebra, permite explicar el surgimiento del Coloquio GeoGebra, organizado por la Comunidad GeoGebra Latinoamericana, como un espacio para impulsar la Educación Abierta en Latinoamérica.

\subsection{Recursos Educativos Abiertos}

Los REA corresponden a creaciones materiales o intelectuales con fines educativos que tienen una licencia libre (Creative Commons), lo cual permite su creación, uso y adaptación por cualquier persona 
sin necesidad de pagar un costo monetario. La definición más extendida de REA es la que propone la UNESCO, según la cual los REA son:

Cualquier recurso educativo (incluso mapas curriculares, materiales de curso, libros de estudio, streaming de videos, aplicaciones multimedia, podcasts y cualquier material que haya sido diseñado para la enseñanza y el aprendizaje) que esté plenamente disponible para ser usado por educadores y estudiantes, sin que haya necesidad de pagar regalías o derechos de licencia (Butcher, Kanwar y Uvalic-Trumbic, 2015, p. 5).

En el caso de GeoGebra, los REA están representados por todas las creaciones que se realizan con su software de matemáticas dinámicas ${ }^{2}$ (también conocidas como applet) o con sus Herramientas de Autor (Actividades ${ }^{3}$, Libros ${ }^{4}$ y Grupos ${ }^{5}$ GeoGebra), ya que todos los recursos así creados tienen por defecto una licencia que dispone de tales recursos de manera libre de cargos para usuarios no comerciales ${ }^{6}$.

Dada la apertura en la creación, el uso y el reuso de los REA, existen de muchos tipos según las características consideradas. Willey, Bliss y McEwen (2014) mencionan que, en términos de los modelos para compartir REA, estos "puede estructurarse y compartirse de diferentes maneras, incluyendo ser compartido como REA individual, compilado y compartido como libros de texto abiertos [open textbooks], y compilado y compartido como cursos abiertos [open courseware]" ( $p$. 783). Estos tres tipos de REA -individual, libro abierto y curso abierto- pueden ser creados con las herramientas ya mencionadas que dispone GeoGebra (software, Actividades, Libros y Grupos), gracias a la articulación que se puede hacer entre ellos. Esto muestra la gran cobertura y posibilidades de creación y uso de REA que permite GeoGebra, lo cual se integra en su repositorio llamado Recursos para el Aula (ver Figura 1).

\footnotetext{
${ }^{2}$ Versión en línea del software de matemáticas dinámicas GeoGebra: https://www.geogebra.org/classic

${ }^{3}$ Tutorial en español sobre cómo crear y usar las Actividades GeoGebra en https://www.geogebra.org/m/K2ekJs69

${ }^{4}$ Tutorial en español sobre cómo crear y usar los Libros GeoGebra en https://www.geogebra.org/m/Smmt4pVM

${ }^{5}$ Tutorial en español sobre cómo crear y usar los Grupos GeoGebra en https://www.geogebra.org/m/Ucar7PHU

${ }^{6}$ Ver la Licencia de GeoGebra en https://www.geogebra.org/license
} 


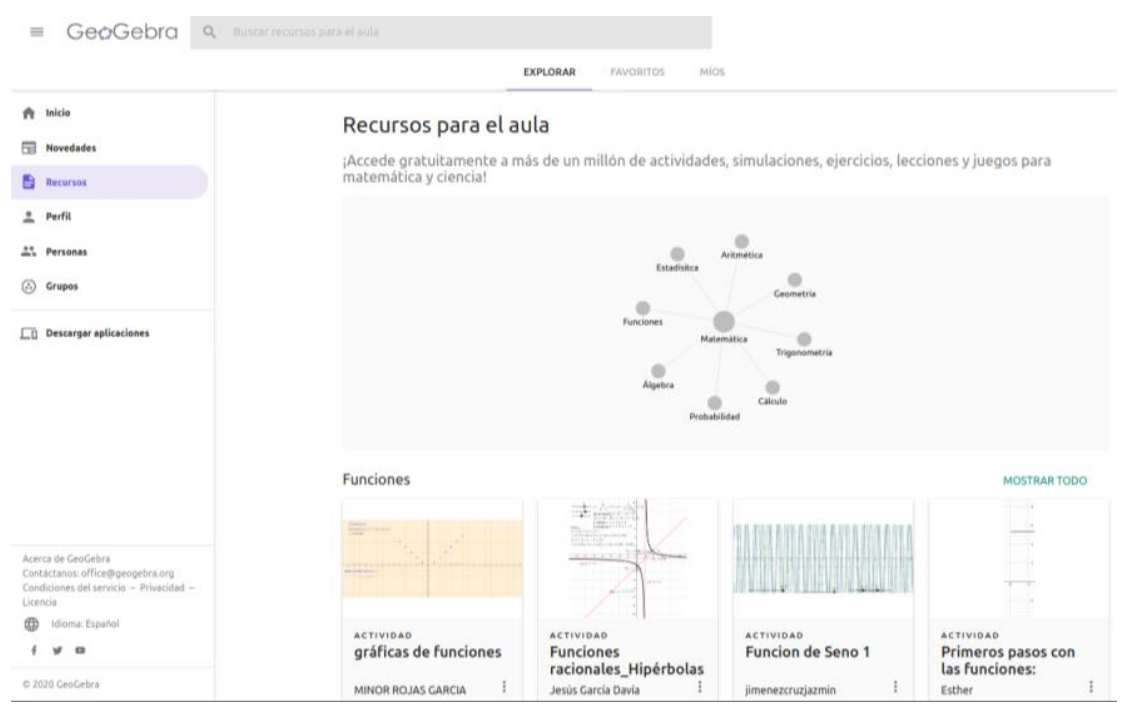

Figura 1: Repositorio de REA de GeoGebra Recursos para el aula. Fuente: Sitio web de GeoGebra (geogebra.org/materials).

\subsection{Prácticas Educativas Abiertas}

Sumado a la disponibilidad tecnológica de herramientas para la creación de REA, un siguiente aspecto importante a considerar es lo que se hace con tales tecnologías y los recursos educativos. A la preocupación por las formas de usar y compartir REA se les conoce en la literatura especializada como Prácticas Educativas Abiertas (PEA). Al respecto, Cronin (2017) propone una definición de PEA a partir de una amplia revisión de literatura especializada que describen o teorizan alguna o todas las PEA:

Prácticas colaborativas que incluyen la creación, el uso y la reutilización de REA, así como prácticas pedagógicas que emplean tecnologías participativas y redes sociales para la interacción, el aprendizaje entre pares, la creación de conocimiento y el empoderamiento de los alumnos (p. 4).

Como ejemplo de estas PEA, específicamente realizadas con GeoGebra, se pueden mencionar los siguientes casos: la creación de REA para el estudio de funciones de variable compleja a través de representaciones gráficas (Ponce Campuzano, 2020), para el estudio de funciones (Abar, 2020) y campos vectoriales (Del Río, 2020), y para el estudio de superficies algebraicas (Moncada Andino, 2020); estrategias pedagógicas para el estudio de monumentos y edificios públicos, mediante el análisis de fotografías de tales edificios y la construcción de modelos matemáticos (Aguilar, 2020), la modelación de fenómenos físicos a través de la experimentación en condiciones reales y el posterior procesamiento de datos con GeoGebra (Villamizar Araque, 2020), y la resolución de problemas geométricos de manera colaborativa por parte de un grupo de profesores (Poveda, 2020); o la construcción de escenarios animados con GeoGebra por parte de estudiantes de primaria, para la construcción de conocimiento sobre funciones (Basniak, 2020). 
Otro ejemplo para destacar respecto de las PEA realizadas por la Comunidad GeoGebra, corresponde a la creación y compartición de REA de manera abierta por profesoras y profesores usuarios de GeoGebra, a través del repositorio Recursos para el aula (ver Figura 1). Es importante resaltar este ejemplo ya que prácticamente la totalidad de estos REA han sido creados y compartidos por miembros de a Comunidad (como profesoras y profesores), y no por el equipo central de GeoGebra, lo cual cobra aún más sentido y genera un gran impacto al considerar que el repositorio aloja más de un millón de REA (GeoGebra, 2017).

\subsection{Comunidades Educativas Abiertas}

Al crear y usar REA, y poner en funcionamientos PEA se va generando un sentido de pertenencia e identidad con las otras personas, lo cual promueve acciones articuladas que tienen impacto a mayor escala, es decir, se va configurando una Comunidad Educativa Abierta (CEA).

Un ejemplo muy ilustrativo de CEA, dado su popularidad a nivel mundial, es el caso de Wikipedia. $\mathrm{Al}$ respecto podemos hacer un ejercicio introductorio: ¿Qué se nos viene a la mente cuando pensamos en Wikipedia? Lo más probable que se nos venga a la mente su sitio web -en el cual más de alguna vez habremos hecho alguna búsqueda- y alguno de sus artículos que hayamos revisado. Un aspecto de Wikipedia que quizá no se nos venga a la mente con tanta facilidad, a pesar de su relevancia, es la manera en que se construye esta enciclopedia digital. Cada artículo de Wikipedia es construido de manera abierta por una red de usuarios registrados en la plataforma, quienes hacen uso del software libre que sostiene técnicamente a Wikipedia.

En la manera en que se construye Wikipedia es posible reconocer aspectos esenciales que constituyen a esta enciclopedia digital: lo tecnológico y lo pragmático. En cuanto a lo tecnológico se encuentran el software y los artículos, donde el primero corresponde a un software libre y los segundos a REA. En cuanto a lo pragmático, se encuentra la manera en que los usuarios se organizan para redactar, revisar y validar los artículos, es decir, las PEA que ponen en funcionamiento los contribuyentes de Wikipedia (Wikimedia Foundation y Concept Hatchery, 2019).

Con base en estos dos aspectos esenciales y la manera en que se relacionan, es posible caracterizar el aspecto social de Wikipedia, es decir, la comunidad. Esta se puede caracterizar en términos antropológico-sociológicos como un conjunto de grupos sociales relevantes que poseen diferentes interpretaciones y objetivos respecto de la tecnología que los reúne (e.g. Wikipedia, GeoGebra, Creative Commons, GNU/Linux, Arduino), aunque todos ellos están aunados por un propósito común que funciona como pegamento social de la comunidad (Rubio-Pizzorno, 2018).

En ese sentido, la comunidad que se configura alrededor de Wikipedia y le da vida a este proyecto corresponde -en su dimensión oficial- a Wikimedia Foundations, junto a los diferentes grupos de voluntarios de la Wikipedia -en su dimensión no oficial-. Cada uno de estos grupos sociales tiene 
diferentes interpretaciones y objetivos respecto de la Wikipedia, como la construcción de la identidad de Wikimedia, el compromiso con los movimientos abiertos, la preservación de un idioma, la diversidad geográfica, la justicia social, entre otros (Wikimedia Foundation y Concept Hatchery, 2019). No obstante, todos estos grupos sociales están aunado por el propósito de crear de manera abierta y colaborativa una enciclopedia multilingüe y de contenido libre que todos pueden editar, lo cual constituye el pegamento social que conforma la Comunidad Educativa Abierta de Wikipedia.

En el caso de GeoGebra, tanto los aspectos tecnológicos (representados por la construcción y desarrollo del software y de las Herramientas de autor) como los pragmáticos (representados por las PEA), son parte fundamental de lo que Rubio-Pizzorno (2018) denomina como la construcción social de GeoGebra y que tiene como objetivo explicar la manera en que la relación entre estos dos aspectos constituye progresivamente la Comunidad Educativa Abierta de GeoGebra.

Por una parte, se considera la manera en que el componente tecnológico se moldea socialmente, es decir, por la influencia de las relaciones sociales y las PEA puestas en funcionamiento. Al respecto RubioPizzorno (2018) reconoce que "GeoGebra es moldeada socialmente produciendo tecnologías digitales abiertas, como los recursos educativos [abiertos] y el software libre en sus diferentes versiones” (p. 64). Por otra parte, se aborda la manera en que el componente pragmático es moldeado tecnológicamente, reportando la manera en que los aspectos tecnológicos influyen en las relaciones sociales y las PEA, donde se puede reconocer la fundación del Instituto GeoGebra Internacional (Hohenwarter y Lavicza, 2011) como un hito importante en el desarrollo de GeoGebra a nivel global, donde el desarrollo tecnológico ha influido en la organización social de la comunidad, a nivel oficial y no oficial.

Esta relación mutuamente constituyente entre los aspectos tecnológicos y pragmáticos van configurando la constitución de la CEA de GeoGebra, cuyo propósito es a atender las necesidades, las inquietudes y las ideas de profesoras y profesores de matemáticas y otras asignaturas, de manera abierta y colaborativa, mediante el desarrollo y disposición de tecnologías libres y REA. A la luz este propósito, es posible dar cuenta de un modelo de construcción social de la Comunidad GeoGebra (ver Figura 2), donde los diferentes grupos sociales plantean sus necesidades, inquietudes e ideas, las cuales se convierten en el sustrato de los proyectos de la comunidad. A medida que se van desarrollando estos proyectos, las necesidades de los miembros de la comunidad van siendo atendidos.

Por lo tanto, la evolución mostrada a lo largo de esta sección en términos de los REA, las PEA y las CEA, ha permitido "demostrar la manera en que los artefactos mismos contienen a la sociedad inmersa en ellos" (Pinch, 2015, pp. 25). De ahí la importancia del modelo de construcción social de GeoGebra. 


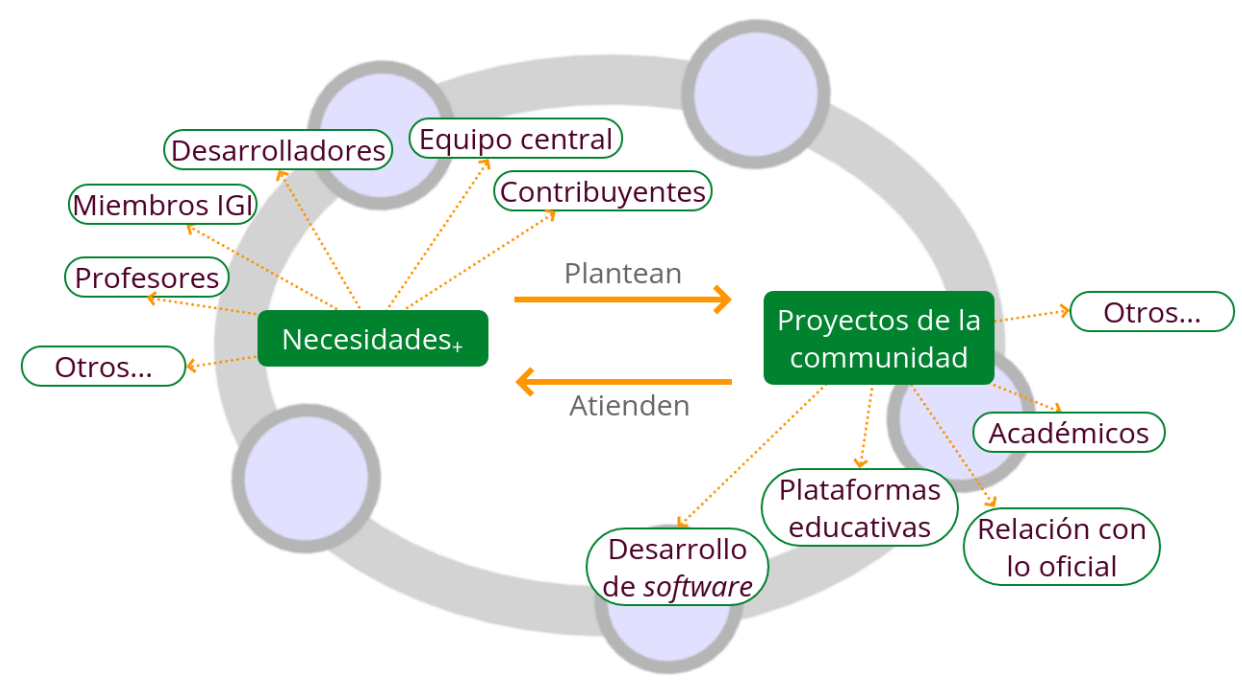

Figura 2: Modelo de construcción social de la Comunidad GeoGebra.

Fuente: (Rubio-Pizzorno, 2018).

\subsection{Comunidad GeoGebra Latinoamericana y su aporte a la Educación Abierta en Latinoamérica}

Luego de revisar la manera en que GeoGebra se ha ido configurando como una CEA con alcance global, a continuación nos centraremos en su funcionamiento en Latinoamérica representado por la Comunidad GeoGebra Latinoamericana (CGL). Para lo cual, en esta sección se presenta una breve descripción cronológica del desarrollo de la CGL.

A partir de 2017, un grupo de entusiastas latinoamericanos de GeoGebra comenzaron a reunirse en un evento académico de la región (la Reunión Latinoamericana de Matemática Educativa) para reflexionar sobre las posibles acciones a realizar para visibilizar los aportes de los miembros de la comunidad GeoGebra de Latinoamérica y articular los esfuerzos de nuestra región (Rubio-Pizzorno et al., 2018). La intención de estos entusiastas es reconocer lo que ya se había hecho en la región y construir con base en estas experiencias. Por ejemplo, Latinoamérica cuenta con 17 institutos GeoGebra locales, con 141 miembros del Instituto GeoGebra Internacional ${ }^{7}$ (IGI), se han realizado cinco ediciones de Congresos Latinoamericanos de GeoGebra (GeoGebra, 2020) y se cuenta con la Revista do Instituto GeoGebra de São Paulo ${ }^{8}$, además del gran aporte que realizan profesoras y profesores al repositorio Recursos para el aula, con la creación y compartición de REA.

No obstante, y a pesar de los esfuerzos ya realizados y de la gran cantidad de miembros del IGI en Latinoamérica, se ha ido diluyendo la fuerza que mostró la comunidad GeoGebra en Latinoamérica hace

\footnotetext{
${ }^{7}$ Según los datos del sitio web https://www.geogebra.org/institutes

${ }^{8}$ Sitio web de la Revista do Instituto GeoGebra Internacional de São Paulo https://revistas.pucsp.br/IGISP/index
} 
algunos años. Muestra de ello es la inactividad de varios miembros latinoamericanos del IGI y de ciertos institutos GeoGebra locales; así también, la no realización del Congreso Latinoamericano de GeoGebra, el cual se llevó a cabo por última vez en 2016. En respuesta a esta situación, el grupo de entusiastas por GeoGebra se comenzó a organizar para realizar acciones que permitieran visibilizar y articular los aportes realizados en la región. Es por ello que el grupo se comenzó a identificar con el nombre de Comunidad GeoGebra Latinoamericana (CGL), por su compromiso con el desarrollo de la Comunidad GeoGebra en y desde Latinoamérica, y con el compromiso de aportar a la Educación Abierta de la región.

Algunos ejemplo de las iniciativas de la CGL son el grupo de discusión Matemática Educativa en la Era Digital realizado en la Reunión Latinoamericana de Matemática Educativa (RELME), donde desde 2017 se han discutido y reflexionado sobre temas relacionado con la CGL, tales como, la visibilización y articulación de la Comunidad GeoGebra Latinoamericana (Rubio-Pizzorno et al., 2018), sobre Recursos Educativos Abiertos creados con GeoGebra, integrando prácticas y tecnologías digitales (Rubio-Pizzorno, León Salinas, García-Cuéllar y Prieto G., 2019) y sobre proyectos de alcance regional realizados por la Comunidad GeoGebra Latinoamericana (Basniak, Sóstenes, García-Cuéllar y Rubio-Pizzorno, en revisión).

Sumados a los anteriores, el proyecto insigne de la CGL corresponde al Coloquio GeoGebra, cuyo objetivo es servir como un espacio para compartir de manera horizontal y reflexionar sistemáticamente, entre interesados en la educación de Latinoamérica (estudiantes, profesores e investigadores). Las reflexiones giran en torno a las formas en que la educación en nuestra región se está relacionando, interactuando y construyendo a propósito de su vínculo con la cultura digital y, específicamente, con GeoGebra. También se apunta a sortear la barrera económica y de movilización en nuestra región, por ello se ha adoptado la modalidad en línea para el Coloquio GeoGebra. Y, sobre todo, centrarnos en nuestras fortalezas de lenguaje común, resiliencia, creatividad y solidaridad, todas ellas características del pueblo latinoamericano, para aportar a la educación en nuestra región desde una perspectiva abierta, es decir, para impulsar la Educación Abierta en Latinoamérica

Los proyectos de la CGL son una muestra de la necesidad de constituir, desarrollar y consolidar acciones de manera comunitaria para impulsar la Educación Abierta en Latinoamérica. Esto permite ampliar y robustecer la definición de la Educación Abierta provista por la Declaración de Panamá (2018), en donde se consideran únicamente los REA y las PEA, a saber: "prácticas y políticas que aseguren el acceso, apertura, modificación y reutilización de Recursos Educativos Abiertos (REAs), datos, metodologías y procesos educativos" (p. 6).

De esta manera, la CGL pretende aportar a la Educación Abierta desde la creación y difusión de REA, el desarrollo e implementación de PEA, y la constitución, desarrollo y consolidación de CEA a través de instancias de visibilización y permanente articulación comunitaria. 


\section{Coloquio GeoGebra}

Luego de haber expuesto el contexto general de este artículo, a saber la Educación Abierta, nos centramos en un proyecto de la CGL -el Coloquio GeoGebra- para ilustrar su propósito de impulsar la Educación Abierta en Latinoamérica.

Este evento tiene su origen en las reflexiones llevadas a cabo por los miembros de la comunidad, en la segunda edición del grupo de discusión de la Relme, Matemática Educativa en la Era Digital, donde se propuso la creación del Coloquio GeoGebra como una instancia que permitiera juntarnos a reflexionar de manera permanente y así no tener que esperar un año a vernos en el grupo de discusión. De esta manera, se propuso que el Coloquio GeoGebra se constituyera como:

Un espacio de reflexión permanente y sistemática, que nos permita compartir a los miembros de la Comunidad nuestras experiencias docentes o de investigación relacionadas con GeoGebra. Este proyecto se planteó durante la segunda edición del Grupo de discusión, con el objetivo de llevarlo a cabo a partir del 2019 (Rubio-Pizzorno et al., 2019, p. 699).

Con esta idea, el equipo de la CGL se dedicó a conseguir los recursos necesarios para asegurar las condiciones que permitieran realizar el evento de manera abierta, gratuita y en línea. Así también, el Coloquio GeoGebra se pensó como una instancia democratizadora del conocimiento, donde todos los miembros de la comunidad (estudiantes, profesores, investigadores y entusiastas de GeoGebra) estuvieran en las mismas condiciones de participar. Así, se configuró el Coloquio GeoGebra para comenzar en el año 2019, con sesiones mensuales, gratuitas, en línea y sin necesidad de registro.

\subsection{Estructura de las sesiones del Coloquio GeoGebra}

La estructura de cada sesión del Coloquio GeoGebra está determinada por los diferentes roles de los participantes: presentador, conferencista, moderador y asistentes:

La presentadora o el presentador tiene la responsabilidad de abrir las sesiones, comunicar los aspectos generales del evento y su desarrollo (como las secciones de la sesión), dar avisos de la CGL, y encargarse de la gestión técnica de la sesión, como por ejemplo: iniciar y finalizar la videollamada, cuidar que sólo estén abiertos los micrófonos del conferencista y la moderadora, apagar las cámaras de los asistentes si se están mostrando imágenes no apropiadas, entre otros.

$>$ La moderadora o el moderador se encarga de la gestión académica de la sesión, es decir, presenta al conferencista leyendo su semblanza y el resumen de la conferencia; indica a los asistentes que pueden realizar preguntas durante el transcurso de toda la conferencia a través del chat de la videollamada, las cuales son leídas al finalizar la conferencia; y gestiona la ronda de preguntas.

$>$ La o el conferencista es quien comparte una experiencia escolar, docente o investigativa con el uso de GeoGebra. Se apoya de una presentación para compartir su conferencia, que usualmente 
tiene una duración entre 45 y 60 minutos; luego de finalizada la conferencia se realiza la ronda de preguntas donde el conferencista responde las inquietudes de las y los asistentes.

> Las y los asistentes a la sesión pueden disfrutar de la presentación del conferencista; hacer preguntas que son respondidas durante la misma sesión; además de estar al tanto de los proyectos relacionados con GeoGebra realizados en Latinoamérica.

Cabe destacar que tanto el presentador como las moderadoras son parte del equipo de la CGL. En específico el rol de presentador ha estado a cargo de Sergio Rubio-Pizzorno (de México y Chile) y Laura del Río (de Argentina), y los y las moderadoras se detallan en la Tabla 1. Además, se tiene como objetivo del evento que las conferencias puedan ser impartidas en español y algunas también en portugués, de manera que estén considerados los dos idiomas oficiales más extendidos en Latinoamérica.

\subsection{Año 1 del Coloquio GeoGebra (2019)}

El primer año del Coloquio GeoGebra se realizó entre marzo y diciembre de 2019, contando con 10 sesiones, cuyos conferencistas representan a siete países diferentes de Latinoamérica, abarcando el norte, el centro y el sur de América (ver Tabla 1): Argentina, Brasil, Colombia, Costa Rica, Ecuador, México, Uruguay. De estas sesiones, dos fueron en portugués y ocho en español.

Como estrategia de lanzamiento del evento, el equipo de la CGL decidió realizar una marcha blanca durante el primer semestre de 2019, correspondiente a las cinco primeras sesiones del Coloquio GeoGebra. En estas sesiones, tanto los moderadores como los conferencistas fueron miembros del equipo de la CGL. La marcha blanca del evento tuvo gran éxito, lo cual se reflejó en la recepción e interés de los miembros de la comunidad. Con base en esto, en el segundo semestre se dio una dinámica propia de las CEA, donde ciertos miembros de la comunidad se pusieron en contacto con el equipo de la CGL, de manera autónoma y por motivación propia, para expresar su interés en compartir una conferencia en el Coloquio GeoGebra, completando así todas las sesiones del segundo semestre de 2019.

En sintonía con el objetivo de abrir la información generada en el Coloquio GeoGebra, además de la transmisión en vivo, en línea y gratuita, todos los videos de las sesiones se publican en el canal de YouTube de la Comunidad GeoGebra Latinoamericana ${ }^{9}$, para que todas y todos puedan acceder a las sesiones en cualquier momento y verlas al ritmo que más se acomode a cada uno. La edición de los videos de las sesiones del Coloquio GeoGebra han sido realizada por Francisco J. Anaya-Puebla (de México), miembro del equipo de la CGL.

\footnotetext{
${ }^{9}$ Canal de YouTube de la Comunidad GeoGebra Latinoamericana: https://www.youtube.com/GeoGebraLatino
} 
Tabla 1: Detalle de las sesiones del Año 1 del Coloquio GeoGebra ${ }^{10}$

\begin{tabular}{|c|c|c|c|c|}
\hline Sesión & \multicolumn{2}{|c|}{ Conferencista } & \multicolumn{2}{|c|}{ Moderador(a) } \\
\hline 1 & Colombia & William Jiménez & 1 Perú & Daysi García-Cuéllar \\
\hline 2 & Argentina & Laura del Río & Colombia & William Jiménez \\
\hline 3 & W'México & Clara Moncada & Argentina & Laura del Río \\
\hline 4 & 吾Uruguay & Gustavo Aguilar & - Panamá & Keila Chacón \\
\hline 5 & ECosta Rica & William Poveda & Colombia & Camilo Súa \\
\hline 6 & Brasil & Maria Ivete Basniak & 1 Perú & Daysi García-Cuéllar \\
\hline 7 & Brasil & Celina Abar & 1 Perú & Daysi García-Cuéllar \\
\hline 8 & Colombia & Freddy Villamizar & Argentina & Mariana Gabriela Torres \\
\hline 9 & s=cuador & Fredy Rivadeneira & Argentina & Karina Rizzo \\
\hline 10 & $\begin{array}{l}\text { México } \\
\text { Australia }\end{array}$ & $\begin{array}{l}\text { Juan Carlos Ponce } \\
\text { Campuzano }\end{array}$ & México & $\begin{array}{l}\text { Francisco J. Anaya- } \\
\text { Puebla }\end{array}$ \\
\hline
\end{tabular}

Fuente: Elaboración propia.

Así también, se dispuso de la creación de un Libro GeoGebra como Memorias del Año 1 del Coloquio GeoGebra ${ }^{11}$, donde se encuentran de manera abierta las imágenes de difusión de cada sesión, la semblanza de la o el conferencista, el resumen de la conferencia, la presentación en PDF, el video íntegro de la sesión, los REA usados durante la conferencia y algunos que recomiende el o la conferencista, y el artículo asociado a la sesión, publicado en el número especial del volumen 9 de la Revista do Instituto GeoGebra Internacional de São Paulo.

\section{Consideraciones finales}

A la luz de lo expuesto en el presente artículo, se puede reconocer al Coloquio GeoGebra como un espacio de articulación y visibilización de los esfuerzos de estudiantes, profesores e investigadores latinoamericanos, aunados por un propósito educativo común. Así también, da muestra de las características propias de los proyectos de una CEA, tales como su constante ajuste a las necesidades de los miembros de la comunidad, y una permanente revisión de lo realizado a través de la participación de los miembros de la comunidad en el proyecto.

Estos elementos dan cuenta de la manera en que las personas se van involucrando en los proyectos, como el Coloquio GeoGebra, lo cual va desarrollando y consolidando la comunidad. Y al ser objetivos y un evento con fines educativos, la Comunidad GeoGebra Latinoamericana se va desarrollando y consolidando como una Comunidad Educativa Abierta. Esta conclusión es muy relevante, ya que representa la importancia de los aspectos sociales a gran escala en el desarrollo de la Educación Abierta

\footnotetext{
${ }^{10}$ Lista de reproducción de todas las sesiones del Año 1 del Coloquio GeoGebra https://bit.ly/ColoquioGeoGebraA1

${ }^{11}$ Las memorias del Año 1 del Coloquio GeoGebra se pueden encontrar en https://www.geogebra.org/m/zavpecwu
} 
en Latinoamérica, los cuales pueden ser de gran ayuda para la definición de políticas públicas de los países de la región.

De esta manera, es razonable ampliar la definición de Educación Abierta en Latinoamérica presentada en la Declaración de Panamá, de una que considera únicamente aspectos técnicos y pragmáticos, a un modelo de desarrollo de la Educación Abierta sustentado en el encuentro y articulación entre aspectos tecnológicos, pragmáticos y comunitarios, específicamente referidos a los Recursos Educativos Abiertos (REA), las Prácticas Educativas Abiertas (PEA) y las Comunidades Educativas Abiertas (CEA) respectivamente. Así se asegura que tanto los ámbitos oficiales y no oficiales relacionados con la Educación Abierta en Latinoamérica estén considerados como fundamentales para su desarrollo y consolidación.

\section{Referencias}

Abar, C. (2020). A Transposição Didática na criação de estratégias para a utilização do GeoGebra. Revista do Instituto GeoGebra Internacional de São Paulo, 9(1), 59-75. http://doi.org/10.23925/22379657.2020.v9i1p59-75

Aguado-López, E., Becerril-García, A. y Chávez-Ávila, S. (2019). Reflexión sobre la publicación académica y el acceso abierto a partir de la experiencia de RedALyC. Palabra Clave (La Plata), 8(2), e067. https://doi.org/10.24215/18539912e067

Aguilar, G. (2020). Modelos en GeoGebra para el plano y el espacio. Impresión de materiales 3D para su uso en el aula. Revista do Instituto GeoGebra Internacional de São Paulo, 9(1), 132-146. http://doi.org/10.23925/2237-9657.2020.v9i1p132-146

Basniak, M. I. (2020). A construção de cenários animados no GeoGebra e o ensino e aaprendizagem de funções. Revista do Instituto GeoGebra Internacional de São Paulo, 9(1), 43-58. http://doi.org/10.23925/2237-9657.2020.v9i1p43-58

Basniak, M. I., Sóstenes, H., García-Cuéllar, D. y Rubio-Pizzorno, S. (en revisión). Matemática Educativa en la Era Digital. Proyectos de alcance regional realizados por la Comunidad GeoGebra Latinoamericana. En Acta Latinoamericana de Matemática Educativa, 33, (en revisión). Ciudad de México, México: Comité Latinoamericano de Matemática Educativa. ISSN: 2448-6469.

Butcher, N., Kanwar, A. y Uvalic-Trumbic, S. (2015). Guía Básica de Recursos Educativos Abiertos (REA). Francia: UNESCO. https://unesdoc.unesco.org/ark:/48223/pf0000232986

Cronin, C. (2017). Openness and Praxis: Exploring the Use of Open Educational Practices in Higher Education. The International Review of Research in Open and Distributed Learning, 18(5), 15-34. https://doi.org/10.19173/irrodl.v18i5.3096 
Del Río, L. (2020). Recursos para la enseñanza del Cálculo basados en GeoGebra. Revista do Instituto GeoGebra Internacional de São Paulo, 9(1), 120-131. http://doi.org/10.23925/22379657.2020.v9i1p120-131

Free Software Foundation (2020). What is free software? https://www.fsf.org/about/what-is-free-software GeoGebra. (2020, 4 de marzo). En Wikipedia. https://es.wikipedia.org/wiki/GeoGebra

GeoGebra [@geogebra]. (2017, 20 de junio).Find over 1 million free and interactive classroom resources on http://geogebra.org/materials. Search for a topic and share! [Tweet]. Twitter. https://twitter.com/geogebra/status/932586021010714624

Hohenwarter, M. y Lavicza, Z. (2011). The Strength of the Community. En L. Bu y R. Schoen (Eds.), Model-Centered Learning, 7-12. Rotterdam: SensePublishers. https://doi.org/10.1007/978-94$\underline{6091-618-2_{2}}$

Moncada Andino, C. (2020). Construcción de superficies no convencionales con GeoGebra 3D. . Revista do Instituto GeoGebra Internacional de São Paulo, 9(1), 90-100. http://doi.org/10.23925/22379657.2020.v9i1p90-100

Pinch, T. (2015). La construcción social de la tecnología: una revisión. En M. J. Santos y R. Díaz Cruz (Eds.), Innovación tecnológica y procesos culturales. Perspectivas teóricas (pp. 18-37). Fondo de Cultura Económica, México. ISBN 9786071622167.

Ponce Campuzano, J. C. (2020). Una introducción al método de dominio colorado con GeoGebra para la visualización y estudio de funciones complejas. Revista do Instituto GeoGebra Internacional de São Paulo, 9(1), 101-119. http://doi.org/10.23925/2237-9657.2020.v9i1p101-119

Poveda, W. (2020). Resolución de problemas matemáticos en GeoGebra. Revista do Instituto GeoGebra Internacional de São Paulo, 9(1), 26-42. http://doi.org/10.23925/2237-9657.2020.v9i1p26-42

Rubio-Pizzorno, S. (2018). Integración digital a la práctica del docente de geometría. Tesis de Maestría no publicada. Ciudad de México, México: Centro de Investigaciones y de Estudios Avanzados (Cinvestav). https://doi.org/10.13140/RG.2.2.15488.94728/1

Rubio-Pizzorno, S., León Salinas, C., León Ríos, J., Córdoba-Gómez, F. y Abar, C. (2018). Matemática Educativa en la Era Digital: Visibilización y Articulación de la Comunidad GeoGebra Latinoamericana. En L. A. Serna y D. Páges (Eds.), Acta Latinoamericana de Matemática Educativa, 31(2), (pp. 1917-1923). Ciudad de México, México: Comité Latinoamericano de Matemática Educativa. ISSN: 2448-6469. https://clame.org.mx/documentos/alme31 2.pdf

Rubio-Pizzorno, S., León Salinas, C., García-Cuéllar, D. y Prieto G., J. L. (2019). Matemática Educativa en la Era Digital: Recursos Educativos Abiertos integrando Prácticas y Tecnologías digitales. En D. J. García-Cuéllar e I. E. Pérez (Eds.), Acta Latinoamericana de Matemática Educativa, 32(2), 
(pp. 1917-1923). Ciudad de México, México: Comité Latinoamericano de Matemática Educativa. ISSN: 2448-6469. https://clame.org.mx/documentos/alme32_2.pdf

Santana, B., Rossini, C. y Pretto, N. D. L. (2013). Recursos Educacionais Abertos: práticas colaborativas políticas públicas. Salvador: EDUFBA; São Paulo: Casa da Cultura Digital. ISBN: 978-85-2321052-6. http://www.aberta.org.br/livrorea/livro/home.html

Santos, A. I. (2013). Educação aberta: histórico, práticas e o contexto dos recursos educacionais abertos. En B. Santana, C. Rossini y N. D. L. Pretto (Eds.), Recursos Educacionais Abertos: práticas colaborativas políticas públicas, (pp. 71-90). Salvador: EDUFBA; São Paulo: Casa da Cultura Digital. ISBN: 978-85-232-1052-6. http://www.aberta.org.br/livrorea/livro/home.html

Stacey, P. y Hinchliff Pearson, S. (2017). Made With Creative Commons. Copenhagen, Dinamarca: Ctrl+Alt+Delete Books. https://creativecommons.org/made-with-cc/

Villamizar Araque, F. (2020). GeoGebra como herramienta mediadora en la modelización de un fenómeno físico. Revista do Instituto GeoGebra Internacional de São Paulo, 9(1), 76-89. http://doi.org/10.23925/2237-9657.2020.v9i1p76-89

Wikimedia Foundation y Concept Hatchery (2019). Las personas que organizan en el movimiento Wikimedia. Comprendiendo a quienes impulsan el movimiento. https://meta.wikimedia.org/wiki/Movement_Organizers

Willey, D., Bliss, T. L. y McEwen, M. (2014). Open Educational Resources: A Review of the Literature. En J. M. Spector, M. D. Merrill, J. Elen y M. J. Bishop (eds.), Handbook of Research on Educational Communications and Technology (pp. 781 - 789). New York: Springer Science+Business Media. ISBN: 978-1-4614-3185-5. https://doi.org/10.1007/978-1-4614-3185-5 63 


\section{Semblanza del autor}

Profesor e investigador, miembro del Instituto GeoGebra Internacional y Director de la Comunidad GeoGebra Latinoamericana. Interesado en la Educación Matemática, la Integración Digital a la Práctica Docente, la Cultura Digital y la Educación Abierta. Productor y conductor del podcast Aula Abierta ${ }^{12}$, una conversación sobre lo digital desde una perspectiva educativa, en colaboración con el Centro de Cultura Digital de la Secretaría de Cultura de México.

\section{Complementos del artículo}

A continuación, se comparte información relacionada con el Año 1 del Coloquio GeoGebra, organizado por la Comunidad GeoGebra Latinoamericana, con la intención que la lectora o el lector pueda complementar la lectura de este artículo con recursos relacionado a este:

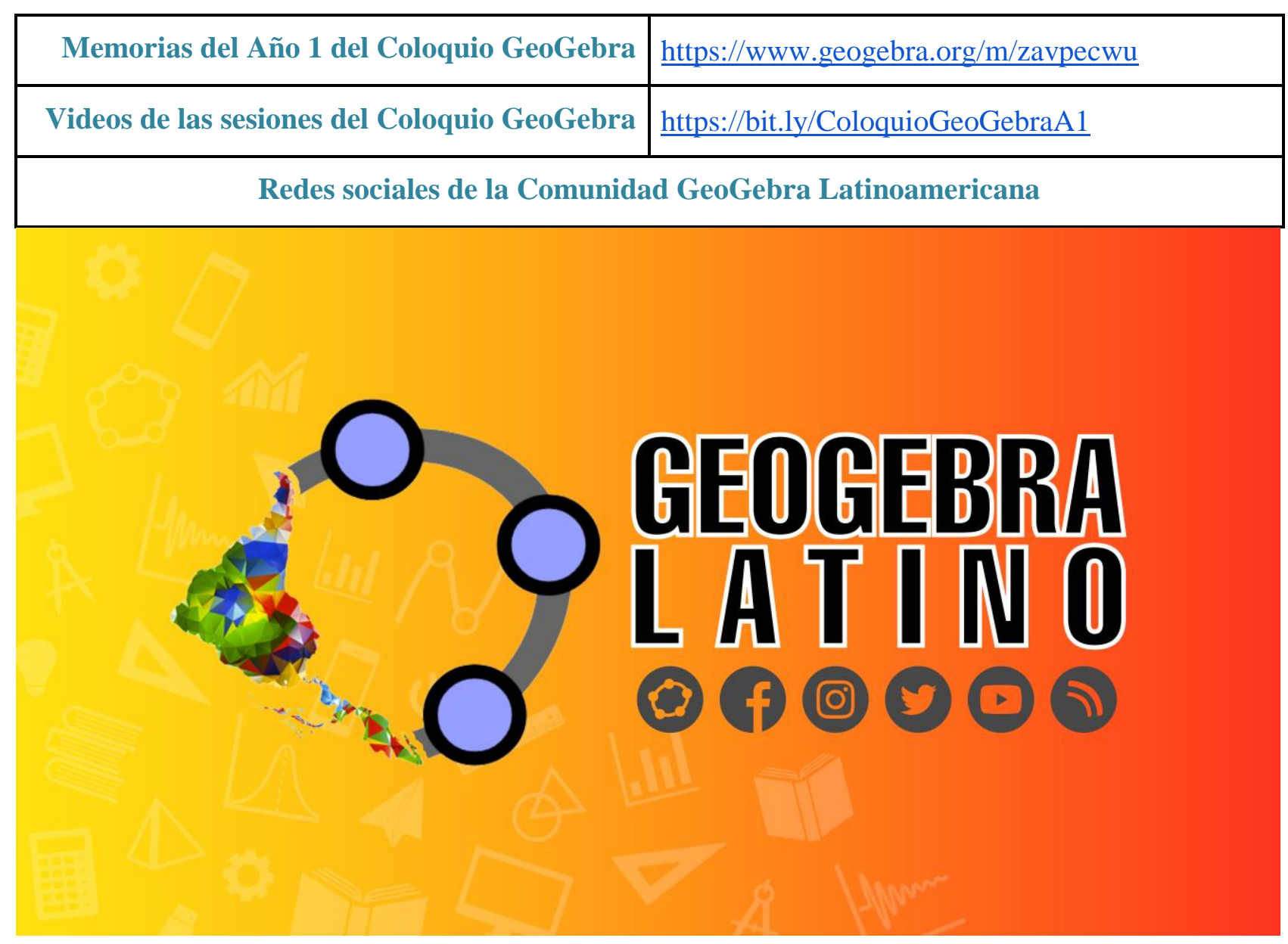

${ }^{12}$ Accede al podcast Aula Abierta a través del siguiente enlace: https://linktr.ee/AulaAbiertaPod 